\title{
In vitro Evidence of Dose-dependent Decrease of Uropathogenic E. coli after Consuming Commercial Cranberry Capsules in Type 2 Diabetic Premenopausal Adult Women: A Literature Review
}

\author{
Dipika Dabas
}

\begin{abstract}
Urinary tract infections (UTIs) arise due to the invasion of pathogenic microorganisms in the urinary tract, resulting in inflammatory reactions in the epithelial linings of the urinary tract. The presence of pathogenic bacteria colonization more than 100,000 microbes of the same strain per milliliter of the urine in two consecutive urine samples is said to be a positive specimen for infection.

An electronic search was conducted by using the PubMed database of the library of Unitec Institute of Technology with key research terms "cranberry," "urinary tract infections," and "type 2 diabetes." These specifications resulted in a number of research studies and review articles, but for this literature review, only random controlled trials, comparative studies, pilot studies, and controlled clinical trials were considered. A total of twelve studies were selected for this review, out of which nine were randomized controlled clinical trials, two were co-relational or observational studies, and one was pilot study. The search was not limited to studies on women; rather, an extensive search was carried out in other subpopulations like children, older age-group people, pregnant women, males, and healthy females that were carried out and published between 2008 and 2014. The random controlled trials in the present review provide level 2 evidence, observational studies provide level 4 evidence, and pilot studies provide level 6 evidence. These levels of evidences are mentioned according to the guidelines provided by Polit and Beck.

Keywords: Cranberry, Premenopausal, Type 2 diabetes, Urinary tract infection.

Journal of Health Sciences \& Research (2020): 10.5005/jp-journals-10042-1094
\end{abstract}

\section{BACKGROUND}

The positive specimen for infection resulting in inflammatory responses in the urinary tract are common and frequent in people suffering from type 2 diabetes besides other infections according to several studies. ${ }^{1-5}$ Various contributing factors that predispose a type 2 diabetic patient to UTI include impairment in the immune system, insufficient metabolic control of diabetes ${ }^{6-9}$, and incomplete emptying of the bladder because of diabetic neuropathy. ${ }^{6,10}$ The classification of UTI can be broadly stated according to the site of infection. ${ }^{11}$

Blood infection in type 2 diabetes can range from asymptomatic bacteriuria to lower urinary tract infection in the form of cystitis, pyelonephritis, etc. Even more serious complications like renal sepsis or necrosis are also observed more frequently in type 2 diabetes compared to other subpopulations. ${ }^{12,13}$ Patients with type 2 diabetes are prone to develop resistant pathogenesis, specially $\beta$-lactamase-positive enterobacteria, carbapenemresistant enterobacteria, etc. The high risk of UTI in type 2 diabetes along with the high rates of antibiotic prescribing further increases the risk of development of resistant strains of urinary pathogens.

\section{Diagnosis and Treatment}

UTIs can be classified according to the mode or route of infection. It can be catheter acquired or community acquired. The other predisposing risk factors contributing to these infections include pregnancy, diabetes mellitus, type 2 diabetes (suppressing the immune system), and hormonal changes during menopause,
Department of Wellness, Healthcare, Nutrition, Research, Medical content Writing, Vipul MedCare, Gurugram, Haryana, India

Corresponding Author: Dipika Dabas, Department of Wellness, Healthcare, Nutrition, Research, Medical content Writing, Vipul MedCare, Gurugram, Haryana, India, Phone: +91 2147483647, e-mail: dipikadabas@gmail.com

How to cite this article: Dabas D. In vitro Evidence of Dose-dependent Decrease of Uropathogenic E. coli after Consuming Commercial Cranberry Capsules in Type 2 Diabetic Premenopausal Adult Women: A Literature Review. J Health Sci Res 2020;11(2):58-63.

Source of support: Nil

Conflict of interest: None

urinary tract abnormalities like enlarged prostate, kidney stones and other factors like use of birth control agents, anatomical characteristics in women like short urethra. Statistical figures are suggested by Schappert and Burt: ${ }^{14}$ One million of the visits in the emergency department, ten thousand of the admission in the hospital, and seven million visits in the clinics are because of community-acquired urinary tract infections.

The UTI prevalence rates in females are also found to be 50 times more than males. ${ }^{15}$ It is suggested that asymptomatic bacteriuria is more common in women because of anatomical characteristics like small size of the urethra and its proximity to the perianal area where there is an abundance of enteric bacteria. ${ }^{16}$ Furthermore, pregnancy also increases susceptibility for these infections, especially in the 6th and 24 th week of gestation ${ }^{17}$ often progressing to complications 
like pyelonephritis leading to outcomes like premature deliveries or low birth weight babies. ${ }^{18}$

According to the Ministry of Health, New Zealand, diabetes is the fastest growing health concern, and together with cardiovascular diseases, it is the main factor contributing to more death every year. The data generated by the Ministry of Health estimate that over 24,000 people in New Zealand are diagnosed with type 2 diabetes and approximately 100,000 people are unaware of this underlining health problem from which they are affected. It is found to be more frequently present in Maori and Pacific Islander populations, where the data show $11.6 \%$ cases in Pacific Islander, $12.4 \%$ cases in South Asian ethnic groups, and $7.8 \%$ in Maori people. The obesity-related type 2 diabetic population is growing every year; it is evident by the data, which suggest an estimate of $42 \%$ of adult Maori and $63.7 \%$ of Pacific Islanders is categorized as obese. The complications related to diabetes like urine infections, neuropathy, and skin infections are one of the leading causes of rise in not only the morbidity and mortality but also pose a burden on the Nation's economy.

An observational study in patients with type 2 diabetes in the UK found UTI incident rates higher in diabetic patients than in other general populations. ${ }^{19}$ An estimate ranging from 8 to $27 \%$ of a symptomatic bacteriuria is reported in type 2 diabetes by many other studies. ${ }^{6,8,20}$ Diabetes is among one of the risk factors for urinary tract infections, others being catheterization, urinary retention intercourse, etc. Many potential mechanisms that are distinct in type 2 diabetes responsible for the increased risk of UTI as suggested by some of the studies include increased level of renal parenchymal glucose, presence of favorable medium for the growth, and multiplication of pathogenic strains. ${ }^{21,22}$ Diabetic women were found to be 6 to 15 times more hospitalized than nondiabetic women according to a Canadian study. In addition to this, according to Freeman et al. ${ }^{23}$ and Williamson et al., ${ }^{24}$ for the high endemicity of extended-spectrum $\beta$-lactamase enterobacteriaceae (ESBL-E) found in New Zealand in community-onset urinary infections, the treatment is posing a great therapeutic challenge. This fact is also confirmed by the data generated by the laboratory, New Zealand in 2005, which described an expenditure of $\$ 12.5$ million for performing urine cultures in the hospitals.

The standard treatment for UTI is the antimicrobial therapy, which includes medicines like amoxicillin, quinolones, and trimethoprim. These empirical medications are not only expensive but also often accompanied by unnecessary side effects. This widespread antibiotic prescribing has raised the concerns globally regarding the development of antibiotic-resistant strains of uropathogens, especially Escherichia coli. Thus, this fact notifies us to consider some non-antibiotic or alternative method to treat or prevent urinary infections.

\section{Treatment with Vaccinum Macrocarpon}

Cranberry and various products derived from it have been used as a complementary and alternative therapy in the prevention and treatment of UTI over the decades. There are many suggested theories explained for its action. In 1920, acidification of the urine after its consumption is considered as its mode of action, whereas Liu et al. ${ }^{25}$ described two of its constituents, i.e., fructose and an unknown polymeric compound with their antibacterial antiadherence activity as a possible way of action. Despite the limited and varied knowledge of the science behind the cranberries action in urine infections, it continues to be famously used in the prophylaxis of these infections.
According to the Cochrane meta-analysis, it was stated that there are evidences of prevention of recurrent UTI in women after cranberry consumption, but it did not mention its effectiveness in other subpopulations. ${ }^{15}$ This shows that though the cranberry prophylaxis is a popular practice in the management of urine infections, the clinical significance in urine infections, especially in a population of type 2 diabetes remain inconclusive. Keeping this in mind, the aim of this review is to examine the literatures and studies since 2008 in order to identify the usefulness of cranberry and its products in the prophylaxis of urine infections, particularly in women population affected with type 2 diabetes. This literature will also try to determine the gaps in other research studies conducted using cranberry products. Jepson and $\mathrm{Craig}^{15}$ conducted a meta-analysis in which an effort was made to systematically combine the results from various studies. It was concluded from this meta-analysis that more large, randomized, double-blind placebo-controlled studies are required in order to identify the mode of action of cranberry product. It also identified the need for finding the optimum dose and mode of its administration for achieving its maximum protective effect. Moreover, previous studies have also shown high dropout rates where cranberry is used as an intervention. All these facts together call for additional evidence-based studies, which can address these gaps.

It is essential to produce evidence-based knowledge to healthcare practitioners like general practitioners, nurses, and pharmacists, who play an integral role in educating and encouraging the valid use of cranberry products in the prophylaxis of urinary tract infections to the patients. Thus, combining the patient's cultural knowledge together with the recent research in clinical practice should be used as a guiding tool in all educational efforts surrounding the use of cranberry or cranberry-based products in the management of UTI.

\section{SynOpsis of the Evidences}

Twelve studies that utilized cranberry juice and capsules in various doses for treatment or prevention of UTIs were reviewed, and mixed results were found. The levels of evidences in the selected studies are mentioned according to the guideline provided by Polit and Beck. ${ }^{3}$ Six studies that utilized cranberry capsules as the intervention: One was an observational study, the other was a pilot study, and the rest four were randomized clinical trials (RCTs). The other six studies that were reviewed used cranberry juice as the intervention and were also randomized control studies. The comparisons in all these studies were made against placebo. The results of these studies indicated positive outcomes (decrease in the urine infection) in trials conducted by McMurdo et al., ${ }^{26}$ while results from Lee et al. ${ }^{27}$ showed no differences in the infection rates. The study of cranberry intervention in older age-group ${ }^{28}$ found the consumption of cranberry useful and effective, especially in those who are at high risk of UTI, whereas Sathosi et al. ${ }^{29}$ did not find any difference in the elderly women population. Studies conducted among children ${ }^{30,31}$ and the result findings with cranberry juice intervention were found to be contraindicatory. The former study showed a positive influence of the intervention in UTIs, whereas the latter showed no significant difference in the first UTI recurrence in children. The intervention of cranberry juice in pregnant women showed positive results in the prevention of UTIs. ${ }^{32}$ The randomized double-blind study ${ }^{33}$ with the intervention of dry form of cranberry in women population also suggested a significant effect of cranberry in those 


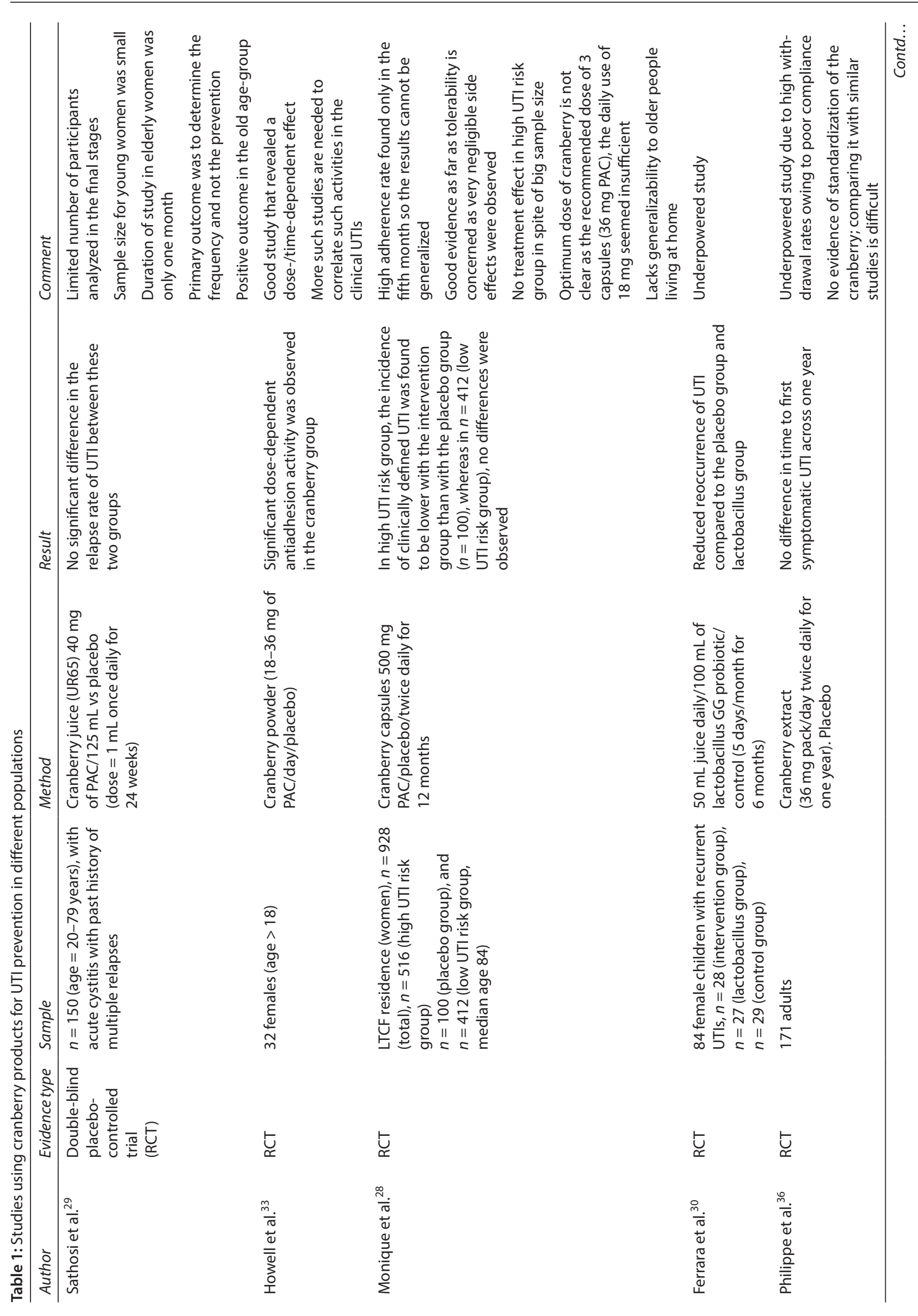




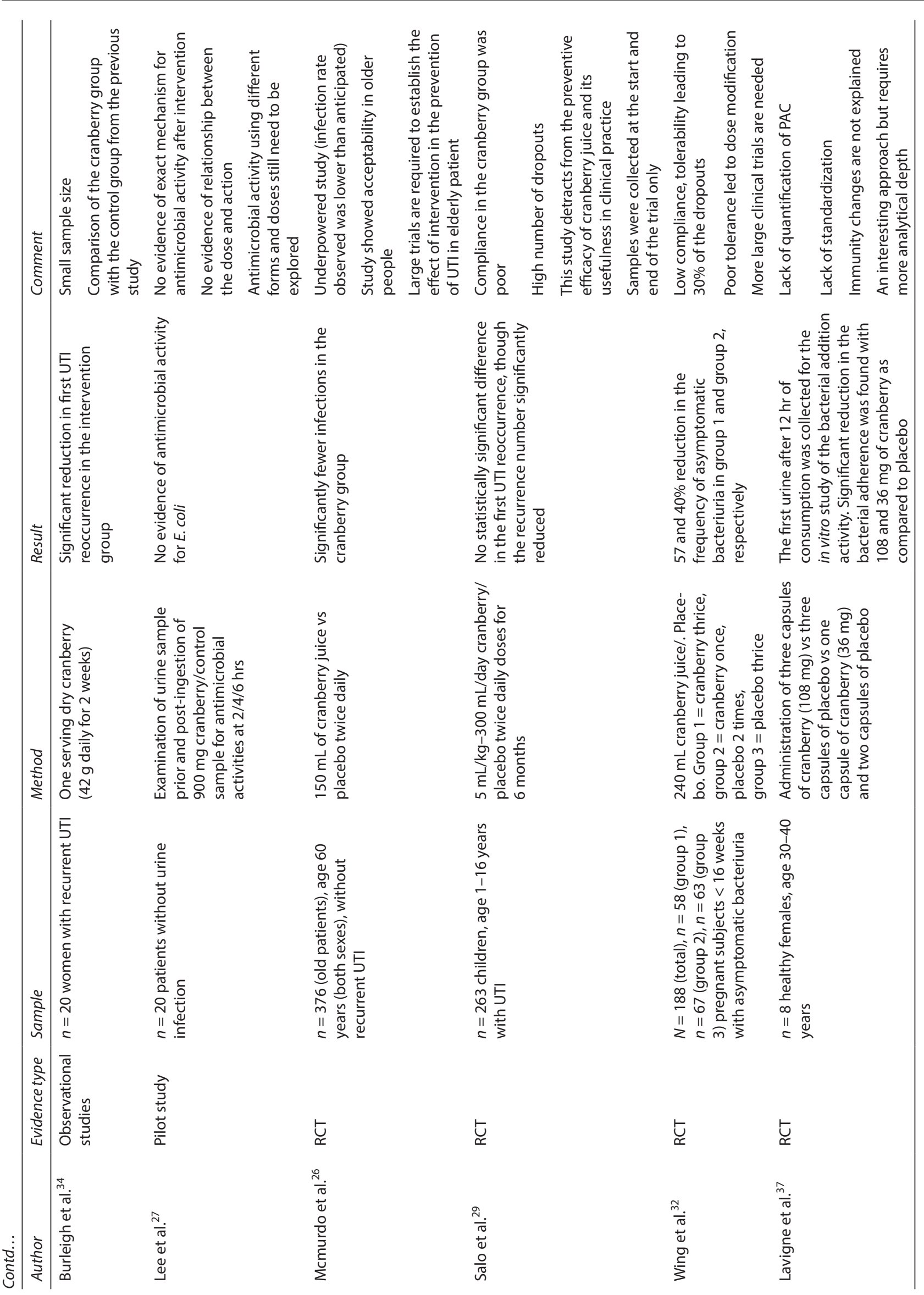


suffering from recurrent UTI, whereas on the contrary, similar population studies in women ${ }^{34}$ did not find any statistical benefit of cranberry in the prevention of UTI. A similar study in women, ${ }^{35}$ but in a different pathology, cervical cancer, found on effect with using cranberry juice as an intervention. The randomized placebo-controlled trial by Phillips et al. ${ }^{36}$ failed to identify the influence of cranberry extract on adults suffering from multiple sclerosis when comparing it to placebo. Only few in vitro studies have been observed in the cranberry interventional studies. One of the special types of in vitro studies by Howell et al. ${ }^{33}$ and by Lavigne et al. ${ }^{37}$ suggested antibacterial antiadhesion activities of cranberry as the potential reason for its use in UTI. One such study clinically proved the bacterial antiadherence properties of cranberry using two different types of doses, whereas another pilot study using similar mechanism showed divergent facts where antimicrobial activities after cranberry consumption were noted only against Klebsiella pneumonia and not against E. coli. ${ }^{37}$ According to Howell et al., ${ }^{33}$ a lower dose of $18 \mathrm{mg}$ was found to be ineffective, whereas a higher dose of $72 \mathrm{mg}$ was found to be more efficient producing $100 \%$ antiadhesion activity. But, this study was conducted on healthy volunteers with no signs and symptoms of urinary infections. The present recommended dose of cranberry, i.e., $500 \mathrm{mg}$ with $36 \mathrm{mg}$ PAC in UTI, is highly arguable as far as PAC concentration and its action on the uropathogens are concerned as evident by the results from these different reviewed studies. The summary of the studies is displayed in Table 1.

\section{SUMMARY}

On reviewing various abovementioned studies, the clear consensus of cranberry and its products for the prevention of UTIs cannot be validated; although majority of the trials (9/12) showed its positive effect, the overall action is still not clear. This might be due to a number of limitations found in these trials like small sample size, high withdrawal rate, short treatment period, insufficient result outcomes to claim generalizability, use of control groups from other previous studies, inclusion of both sexes, lack of standardization of cranberry products making it difficult to determine the optimal therapeutic dose, no clarity on the mode of administration, and improper blinding reporting of the adversarial effects on insensitive groups.

There is no evidence-based study found in determining the efficacy of cranberry products and urine infections in women affected with type 2 diabetes. The evidences regarding the potential effectiveness of cranberry products in UTI prophylaxis or treatment in women population are also found to be arguable. Even the in vitro studies are found to be also limited. Thus, in order to make clinical decisions, more dose-dependent evidence-based large-scale trials are needed. There is much-needed requirement of conducting a trial, which not only suggests a potential mechanism of the action of its constituents like proanthocyanidins but also gives an idea of its concentration to be effectively used, especially in these groups of infections.

\section{Inferences for Practice}

While discussing the management options with females who are prediagnosed or unknowingly suffer from urinary infections as a complication of type 2 diabetes, healthcare providers can recommend cranberry or its products as an alternative or a complimentary prophylactic product, supporting this recommendation with the evidences, which provides a positive result and no other serious adversarial outcomes. However, the treatment should not be replaced with antibiotics until an effective dose, type, and mode of administration are clearly confirmed by further trials; therefore, till that time, it is imperative to consider the patients' personal beliefs and preferences for the treatment so that the best healthcare benefit can be provided in the interest of the sufferers.

\section{References}

1. Shankel S. Symptoms and signs. In Bacterial urinary tract infections. Retrieved from http://www.merckmanuals.com/professional/ genitor-urinary-disorders/urinary_tractinfections_uti/bacterial_ urinary tract_infections. html \# v 1052 851; 2007.

2. Nicolle LE, Bradley S, Colgan R, et al. Infectious Diseases Society of America guidelines for the diagnosis and treatment of asymptomatic bacteriuria in adults. Clin Infect Dis 2005;40(5):643-654. DOI: 10.1086/427507

3. Polit DF, Beck CT. Nursing research: Generating and assessing evidence for nursing practice (9th ed.). Philadelphia: Lippincott, Williams \& Wilkins; 2012.

4. Patterson JE, Andriole VT. Bacterial urinary tract infections in diabetes. Infect Dis Clin North Am 1997;11(3):735-750. DOI: 10.1016/s08915520(05)70383-4.

5. Joshi N, Caputo GM, Weitekamp MR, et al. Infections in patients with diabetes mellitus. N Engl J Med 1999;341(25):1906-1912. DOI: 10.1056/ NEJM199912163412507.

6. Fünfstück R, Nicolle $L E$, Hanefeld $M$, et al. Urinary tract infection in patients with diabetes mellitus. Clin Nephrol 2012;77(1):40-48. DOI: $10.5414 / \mathrm{cn} 107216$.

7. Geerlings SE, Stolk RP, Camps MJ, et al. Asymptomatic bacteriuria can be considered a diabetic complication in women with diabetes mellitus. Adv Exp Med Biol 2000;485:309-314. DOI: 10.1007/0-30646840-9_41.

8. Truzzi JC, Almeida FM, Nunes EC, et al. Residual urinary volume and urinary tract infection-when are they linked? J Urol 2008;180(1):182-185. DOI: 10.1016/j.juro.2008.03.044.

9. Valerius $\mathrm{NH}$, Eff $\mathrm{C}$, Hansen $\mathrm{NE}$, et al. Neutrophil and lymphocyte function in patients with diabetes mellitus. Acta Med Scand 1982;211(6):463-467. DOI: 10.1111/j.0954-6820.1982.tb01983.x.

10. Hosking DJ, Bennett T, Hampton JR. Diabetic autonomic neuropathy. Diabetes 1978;27(10):1043-1055. DOI: 10.2337/diab.27.10.1043.

11. Jancel T, Dudas V. Management of uncomplicated urinary tract infections. West J Med 2002;176(1):51-55. DOI: 10.1136/ewjm.176.1.5.

12. Brown JS, Wessells $\mathrm{H}$, Chancellor MB, et al. Urologic complications of diabetes. Diabetes Care 2005;28(1):177-185. DOI: 10.2337/ diacare.28.1.177.

13. Kofteridis DP, Papadimitraki E, Mantadakis E, et al. Effect of diabetes mellitus on the clinical and microbiological features of hospitalized elderly patients with acute pyelonephritis. J Am Geriatr Soc 2009;57(11):2125-2128. DOI: 10.1111/j.1532-5415.2009.02550.x.

14. Schappert S, Burt C. Ambulatory care visits to physician offices, hospital outpatient departments, and emergency departments: United States, 1997. National Centre for Health Statistics. Vital Health Stat 1999;13(143):1-39.

15. Jepson R, Craig J. Cranberries for preventing urinary tract infections. Cochrane Database Syst Rev 2012;10(10):CD001321. DOI: 10.1002/14651858.CD001321.pub5.

16. Colgan R, Nicolle LE, McGlone A, et al. Asymptomatic bacteriuria in adults. Am Fam Physician 2006;74(6):985-990.

17. Delzell JE, Lefevre ML. Urinary tract infections during pregnancy. Am Fam Physician 2000;61(3):713-720.

18. Gilstrap LC, Leveno KJ, Cunningham FG, et al. Renal infection and pregnancy outcome. Am J Obstet Gynecol 1981;141(6):709-716. DOI: 10.1016/s0002-9378(15)33316-0.

19. Hirji I, Guo Z, Andersson SW, et al. Incidence of urinary tract infection among patients with Type-2 diabetes in the UK General Practice Research Database (GPRD). J Diabetes Complications 2012;26(6):513-516. DOI: 10.1016/j.jdiacomp.2012.06.008. 
20. Zhanel GG, Nicolle LE, Harding GK. Prevalence of asymptomatic bacteriuria and associated host factors in women with diabetes mellitus. The Manitoba Diabetic Urinary Infection Study Group. Clin Infect Dis 1995;21(2):316-322. DOI: 10.1093/clinids/21.2.316.

21. Schneeberger C, Kazemier BM, Geerlings SE. Asymptomatic bacteriuria and urinary tract infections in special patient groups: women with diabetes mellitus and pregnant women. Curr Opin Infect Dis 2014;27(1):108-114. DOI: 10.1097/QCO.0000000000000028.

22. Soo Park B, Lee SJ, Wha Kim Y, et al. Outcome of nephrectomy and kidney-preserving procedures for the treatment of emphysematous pyelonephritis. Scand J Urol Nephrol 2006;40(4):332-338. DOI: 10.1080/00365590600794902.

23. Freeman JT, Rubin J, McAuliffe GN, et al. Differences in risk-factor profiles between patients with ESBL-producing Escherichia coli and Klebsiella pneumoniae: a multicentre case-case comparison study. Antimicrob Resist Infect Control 2014;3:27. DOI: 10.1186/20472994-3-27.

24. Williamson DA, Lim A, Thomas MG, et al. Incidence, trends and demographics of Staphylococcus aureus infections in Auckland, New Zealand, 2001-2011. BMC Infect Dis 2013;13:569. DOI: 10.1186/14712334-13-569.

25. Liu Y, Black MA, Caron L, et al. Role of cranberry juice on molecularsurface characteristics and adhesion behavior of Escherichia coli. Biotechnol Bioeng 2006;93(2):297-305. DOI: 10.1002/bit.20675.

26. McMurdo M, Argo I, Phillips G, et al. Cranberry or trimethoprim for the prevention of recurrent urinary tract infections? A randomized controlled trial in older women. J Antimicrob Chemother 2009;63(2):389-395. DOI: 10.1093/jac/dkn489.

27. Lee $Y L$, Najm W, Owens J, et al. Antimicrobial activity of urine after ingestion of cranberry: A pilot study. Evid Based Complement Alternat Med 2010 Jun;7(2):227-232. DOI: 10.1093/ecam/nem183.

28. Monique AAC, Hout WB, Putter $\mathrm{H}$, et al. Effectiveness of cranberry capsules to prevent urinary tract infections in vulnerable older persons: a double-blind randomized placebo-controlled trial in long-term care facilities. J Am Geriatr Soc 2014 Jan;62(1):103-110. DOI: 10.1111/jgs.12593.

29. Satoshi T, Ryoichi H, Mitsuru $Y$, et al. A randomized clinical trial to evaluate the preventive effect of cranberry juice (UR65) for patients with recurrent urinary tract infection. J Infect Chemother 2013 Feb;19(1):112-117. DOI: 10.1007/s10156-012-0467-7.

30. Ferrara P, Romaniello L, Vitelli $\mathrm{O}$, et al. Cranberry juice for the prevention of recurrent urinary tract infections: a randomized controlled trial in children. Scand J Urol Nephrol 2009;43(5):369-372. DOI: 10.3109/00365590902936698.

31. Salo J, Unari M, Helminen $\mathrm{M}$, et al. Cranberry juice for the prevention of recurrences of urinary tract infections in children: a randomized placebo-controlled trial. Clin Infect Dis 2012 Feb;54(3):340-346. DOI: 10.1093/cid/cir801.

32. Wing D, Rumney P, Preslicka $C$, et al. Daily cranberry juice for the prevention of asymptomatic bacteriuria in pregnancy: A randomized, controlled pilot study. J Urol 2008;180(4):1367-1372. DOI: 10.1016/j. juro.2008.06.016.

33. Howell AM, Botto $H$, Combesure $C$, et al. Dosage effect on uropathogenic Escherichia coli anti-adhesion activity in urine following consumption of cranberry powder standardized for proanthocyanidin content: a multicentric randomized double blind study. BMC Infect Dis 2010;10(1):94-104. DOI: 10.1186/1471-2334-10-94.

34. Burleigh AE, Benck SM, McAchran SE, et al. Consumption of sweetened, dried cranberries may reduce UTI incidence in susceptible women: a modified observational study. Nutr J 2013;12(1):139. DOI: 10.1186/1475-2891-12-139.

35. Cowan CC, Hutchison C, Cole T, et al. A randomised double-blind placebo-controlled trial to determine the effect of cranberry juice on decreasing the incidence of urinary symptoms and urinary tract infections in patients undergoing radiotherapy for cancer of the bladder or cervix. Clin Oncol (R Coll Radiol) 2012;24(2):e31-38. DOI: 10.1016/j.clon.2011.05.009.

36. Phillips G, Gerard A, Nicolas B, et al. Cranberry versus placebo in the prevention of urinary infections in multiple sclerosis: a multicenter, randomized, placebo-controlled, double-blind trial. Mult Scler 2014 Aug;20(9):1252-1259. DOI: 10.1177/1352458513517592.

37. Lavigne JP, Bourg G, Combesaue C, et al. In-vitro and in-vivo dosedependent decrease of uropathogenic Escherichia coli virulence after consumption of commercial Vaccinum macrocarpon (cranberry) capsules. Clin Microbiol Infect 2008 Apr;14(4):350-355. DOI: 10.1111/j.1469-0691.2007.01917.x. 